\title{
KNOWLEDGE COMPARISON BETWEEN GROUP CHILDCARE CENTRES AND FAMILY CHILDCARES ON SANITATION OF TOYS
}

\author{
by \\ Yun Ha (Sammie) Hwang \\ Bachelor of Technology, British Columbia Institute of Technology, 2017
}

PROJECT SUBMITTED IN PARTIAL FULFILLMENT OF

THE REQUIREMENTS FOR THE DEGREE OF

Bachelor of Technology in Environmental Health

(C) Yun Ha (Sammie) Hwang

BRITISH COLUMBIA INSTITUTE OF TECHNOLOGY

April 2017

All rights reserved. This work may not be reproduced in a whole or in part, by photocopy or other means, without permission of the author 
The views expressed in this paper are those of the author and do not necessarily reflect the official policy, position or views of BCIT, the Environmental Health program or its faculty. 


\title{
Knowledge comparison between group childcare centers and family childcares on sanitation of toys
}

\author{
Yun Ha (Sammie) Hwang ${ }^{1}$, Helen Heacock ${ }^{2}$, Fred Shaw ${ }^{3}$ \\ 1 Lead Author, B. Tech student, School of Health Sciences, British Columbia, Institute of Technology, 3700 Willingdon Ave, Burnaby, BC V5G 3H2 \\ 2 Supervisor, School of Health Sciences, British Columbia Institute of Technology, 3700 Willingdon Ave, Burnaby, BC V5G 3H2 \\ 3 Contributor, School of Health Sciences, British Columbia Institute of Technology, 3700 Willingdon Ave, Burnaby, BC V5G 3H2
}

\section{Abstract}

Background: Childcare facilities (CCFs) are known to have a high potential risk of exposure and transmission of infectious diseases through contact surfaces, such as toys. Research to date suggests that toys are a potential source of cross-infections in CCFs, especially when childcare providers do not practice proper hygiene. Currently, there is a lack of knowledge on the differences in sanitation methods of toys between group and family CCFs. This study compared knowledge of group and family CCFs regarding how to sanitize toys.

Methods: Self-administered surveys were distributed to group and family CCFs in Surrey, BC via e-mail. The survey was used to assess the knowledge of childcare providers on sanitation of toys. The survey was evaluated using a scoring system. In addition, each participant answered descriptive questions, such as the existence of sanitation plans and toy cleaning and sanitizing schedules.

Results: Group and family CCFs showed no statistically significant differences in knowledge levels on sanitation of toys. The mean score of the knowledge level of group and family CCFs was $65 \%$ and $55 \%$ respectively.

Conclusion: Childcare providers in CCFs play a key role in properly sanitizing toys and preventing transmission of infectious diseases between children. Recognizing knowledge gaps in sanitation can lead to policy development as well as improved educational programs.

Key words: childcare, group, family, children, toys, sanitation, public health, knowledge

\section{Introduction}

In 2003, the National Longitudinal Survey of Children and Youth indicated that $54 \%$ of Canadian children aged six months to five years were in childcare facilities (CCFs). Some examples of CCFs include group childcare centers and family childcares. Approximately $60 \%$ of these children were in CCFs on a full-time basis, spending an average of 30 hours per week (1). The need for childcare has increased over time and therefore, more Canadian children are predicted to attend and spend a significant portion of their time in a CCF.

Research from the United States and the Netherlands repeatedly show that children who regularly attend CCFs have a higher risk of being exposed to bacterial and viral pathogens compared to children who stay at home (2). The risk of exposure and transmission of infectious diseases is high in $\mathrm{CCFs}$, because children come from environments with various hygienic practices. This may account for children bringing in microbes or diseases into CCFs. Children also tend to touch many contact surfaces, share toys, and stay in close proximity of each other (3). Therefore, the risk of microbial contamination of contact surfaces and disease transmission through fomites, such as toys, is especially high within a CCF (4). This risk increases even higher if childcare providers do not utilize proper cleaning and disinfecting methods.

Childcare providers should recognize that the facility could be a potential reservoir for transmission of infectious diseases between children. Children are considered one of the high-risk groups in public health as they have immature immune systems and are more susceptible to disease (3). Therefore, childcare providers have the important role of implementing cleaning and disinfecting strategies to reduce and prevent illness occurrences. These strategies may vary between larger CCFs and family childcares based on their knowledge of the potential risks present in CCFs.

The purpose of this review is to investigate the current literature demonstrating the relationship between infectious diseases transmitted through toys and hygienic practices of childcare providers. This review will also differentiate between practices of larger and family CCFs. It will help determine gaps in research, policy, and knowledge, which are valuable to identify key policy or educational implications for CCFs. 


\section{Literature review}

The following subsections will discuss the results of the literature review investigation. The potential infectious diseases in CCFs associated with toys and the factors that increase the transmission of the infectious diseases will be discussed. This subsection is followed by a discussion of the hygienic practices that CCFs utilize to prevent the infectious diseases is described. In addition, a brief comparison between group and family CCFs is provided. To conclude this section, the strengths and limitations of the literature review are discussed.

\section{Potential infectious diseases in CCFs}

CCFs provide numerous opportunities for children to acquire an infectious disease. Infectious diseases in the facilities can be spread directly or indirectly. Methods of direct transmission include person-to-person contact between children or childcare providers. Diseases can also be spread indirectly via contaminated contact surfaces and fomites, such as toys, and food or water $(5,6)$. Previous studies have indicated that infections transmitted in CCFs are mainly respiratory and enteric diseases $(3,7)$.

Viruses are usually the cause of respiratory diseases, such as acute upper respiratory tract infections (3). These viruses may be found on toys due to sneezing, coughing, and direct contact. Studies state that respiratory viruses, such as coronavirus, adenovirus, and bocavirus, are prevalent in both external environments and CCFs (7). Another study confirms that these viruses are present in throats of children affected by respiratory tract infections (8).

Various studies that sampled for bacteria and viruses identified enteropathogens on contact surfaces within childcare facilities $(2,3,5-7,9,10)$. These are spread through the fecal-oral route and cause diarrhea in children. Children often place shared toys directly into their mouths and if toys were contaminated with enteropathogens, it would likely lead to diarrheal infections (5). Van et al. (5) and Pickering et al. (11) have shown that fecal contamination of toys via hands of childcare providers and children increases during diarrheal outbreaks. Another study showed that a CCF with a rotavirus outbreak had approximately $39 \%$ of the toy balls contaminated with rotavirus, an enteropathogen
(12). These studies demonstrate the potential of cross-infection through toys within CCFs.

\section{Factors that increase the transmission of infectious diseases}

Numerous opportunities exist in CCFs for children to acquire infectious diseases. These opportunities are facilitated by various factors that increase the potential of transmission of infectious diseases. Children in CCFs always share the same space and are physically close to each other throughout the day. Therefore, they are frequently in contact with other children, childcare providers, and objects like toys, which allow diseases to spread in the environment (5).

Toys are known to be "fomites with the highest pathogen load" (7) and can have an important role in indirect transmission of disease. A microbiological study conducted by Lee et al. (10) showed that it is mainly human-associated bacteria that contaminate toys, which proposes that enteropathogens can be easily circulated in CCFs. This correlates with the study mentioned previously, which indicated how toys were significantly contaminated with rotavirus during an outbreak (12).

Childcare providers can also indirectly transmit disease by routine activities, which include handling food and inanimate objects and changing diapers. As children lack the knowledge of hygiene, they freely secrete bodily fluids, such as saliva. It is the childcare providers' duty to clean children and any bodily secretions. Lee and colleagues (10) showed that diaper changes accounted for significant distributions of bacterial contamination in CCFs. Previous studies have also reported high numbers of fecal coliforms on childcare provider hands, which reinforces that childcare providers can be critical source of contamination $(13,14)$.

The analyses of these factors indicate that if proper hygienic practices, such as cleaning toys and regular hand washing, are followed by childcare providers, it can reduce the transmission of diseases in CCFs.

\section{Hygienic practices for prevention}

In Canada, there is currently no legislation regulating the sanitation of CCFs. Some provinces have guidelines developed by health authorities on how to clean and sanitize CCFs, but they are mainly 
used as recommendations. Some recommendations include cleaning and sanitizing diaper tables between use and using gloves when handling bodily fluids. Two recommendations that are significant in controlling infectious disease transmission include rigorous hand washing and regular cleaning of contact surfaces and objects, such as toys.

Many researchers agree that hand washing is notably the most important intervention to prevent the spread of diseases $(3,13,9,15,16)$. Hand washing is critical after handling children, changing diapers, using the washroom, and touching clothing or objects that are contaminated with bodily fluids. Hands should always be washed before and after handling food as well.

It is optimal for childcare providers to clean contact surfaces and objects as a daily routine. Previous studies indicate that daily cleaning of toys and thorough cleaning of toys during an outbreak reduces the likelihood of the presence of enteropathogens in CCFs $(2,17,18)$. Little and Cutcliffe (4) also agree that outbreaks in CCFs may be linked to the poor hygienic condition of toys. However, Ibfelt et al. (7) showed that regularly cleaning toys did not have an effect on preventing infections. This may be due to the nature of the study, as they measured "the isolated effect of cleaning toys on infectious diseases" (7), whereas other studies combined the effect of cleaning toys with other interventions such as hand washing. Ibfelt et al. (7) concludes that hand hygiene and excluding sick children from CCFs are likely the most important control measures.

Most research supports the need of basic sanitation in CCFs in order to prevent infectious diseases. Due to the lack of sanitation standards of CCFs, childcare providers are allowed to adopt cleaning policies based on their own knowledge. Ultimately, it is the responsibility of childcare providers to maintain a clean, sanitary environment for children.

\section{Comparison of CCFs}

There are various types of CCFs, including group and family childcares. CCFs are also categorized according to age groups and the maximum number of children that are allowed in a group (19). For the purpose of this literature review, group childcare centers and family childcares will be discussed.
Group childcare centers are public facilities that care for preschool children (30 months to school age) and are allowed to have a maximum of 25 children in each group. Family childcares care for children of various ages and are allowed to have a maximum of 7 children in each group. Family childcares are based in private residences and provide a home-like environment (19).

A community care facility license is required for CCFs that provide care for more than 3 children that are not related to the operator. CCFs in British Columbia, Canada are licensed according to the Child Care Licensing Regulation (19) under the Community Care and Assisted Living Act (20). The regulation entails the "minimum health, safety, and care standards that must be met by all licensed CCFs" (20).

In the Child Care Licensing Regulation (19), Section 46 lists the requirements for health and safety. The licensee must develop a program that follows and teaches children health and hygiene. It also requires the licensee to ensure that any food contact surface is not used for changing diapers. The regulation is outcome-based and therefore, childcare providers are responsible for carrying out their own sanitation plan to maintain a hygienic environment.

In the United States, Sauver et al. (16) carried out a study that compared two home-based childcares, family and group daycare homes, on hygienic practices and the prevalence of acute respiratory illnesses. Results of the study reported that group daycare homes had fewer cases of respiratory illnesses. They hypothesized that group daycare providers were likely to be more educated and trained than family daycare providers, which would make them more "aware of illness-prevention strategies than family day care providers" (16). However, in the United States, home-based day cares have minimal licensing requirements, whereas in Canada, many home-based family daycares are licensed under the legislation. There is also a lack of information about the differences of hygienic practices between these two types of childcares in recent literature.

\section{Strengths and limitations}

Several limitations were present in the studies used to support this literature review. Due to the lack of research in this area, studies date back to 1986. Many studies of this kind are not conducted in 
Canada, but mostly in the United States and Europe. The CCFs in which the studies were conducted may operate and be regulated differently. As such, comparisons between the studies may prove to be difficult.

The studies also had varying sample sizes, where the majority of the studies had relatively small sample sizes. For example, one study sampled from four CCF classrooms (10) and another study had a sample size of 37 (13). This makes it challenging to have statistically significant results and generalize them for conclusions.

In addition, for each study, CCFs that were chosen to participate may have had potential selection bias. CCFs that are more confident in their compliance to hygienic practices may have shown more interest to participate. There also may have been an observer effect, where the childcare providers may have altered their normal behavior, due to awareness of being watched.

\section{Gaps in research, policy, and knowledge}

Recent studies have defined various risk factors in $\mathrm{CCFs}$ that are associated with infectious diseases. However, there is limited research dedicated towards toys as a potential source of cross-infection. There is also a lack of knowledge on the differences in sanitation methods of contact surfaces, especially toys, between group childcare centers and homebased family childcares. The absence of legislation regulating sanitation procedures can lead to the nonstandardized sanitation practices between different CCFs. A survey that compares sanitation procedures between group childcare centers and home-based family childcares can help recognize any insufficient knowledge on maintaining clean contact surfaces. Results may possibly lead to recommendations for advanced training programs for facilities that show knowledge deficiency. The purpose of this research project was to define any knowledge gaps in sanitizing toys between group and family CCFs using a survey. The study also determined if any other knowledge gaps existed in sanitation methods for maintaining CCFs.

\section{Methods}

An online survey was designed to assess the knowledge of sanitation of toys in childcare providers. The survey was also designed so that the participant could complete the survey in approximately 2-3 minutes. The questions were formulated based on the Guidelines for Cleaning Toys in a Child Care Centre or School from a resource book for caregivers and parents designed by Vancouver Coastal Health (21).

From Fraser Health's Public List of Licensed Child Care Facilities list, only group childcares and family childcares located in Surrey were selected (22). All three types of group childcares in Surrey, under 36 months, 30 months to school age, and school age, were combined to result in the total number of 80 . The total number of family childcares in Surrey was 101. Both group and family childcares were all contacted by phone without bias.

The survey was distributed by e-mail and selfadministered. A standardized written dialogue was sent with the consent form and survey in the e-mail to explain the purpose and any benefits or risks in participation. This also included assurance of confidentiality and ethical approval. Contact information was also provided to participants that would allow them to obtain study results.

Descriptive data was analyzed using Microsoft Excel to assess the proportion of group and family CCFs who have sanitation plans, who offer soft toys, and who clean toys at specific intervals. Data collected regarding the knowledge of sanitation of toys was analyzed using Number Crunching Statistical System (NCSS) to determine the difference between group and family childcares.

\section{Inclusion and exclusion criteria}

Any group or family CCF in Surrey licensed with Fraser Health was eligible to participate in this study. Any other types of CCFs, CCFs in regions outside of Surrey, CCFs that do not have sharable toys for children's use, and CCFs that did not provide an email address for participation were excluded from this study.

\section{Ethical considerations}

Each participant was given a description of the nature of the study, benefits and burdens, any procedures involved, and how the data will be used. They were informed that participation was voluntary, that participation could be withdrawn at any point in the study without penalty, and that confidentiality was guaranteed (23). The study was designed to 
cause no potential harm, discomfort, or risk. All participants were provided the researcher's name and contact information that they could use to review the results of study or ask any additional questions.

\section{Results}

This study collected both nominal and numeric data. Nominal data was collected as counts for descriptive statistics. Pie charts are used to show the relative proportion of each answer chosen by both group and family childcare facilities. A scoring system was used to collect numeric data for inferential statistics and an independent samples t-test was performed to compare the difference in knowledge of group and family childcare providers on sanitation of toys.

A total of 16 group CCFs and 29 family CCFs participated in the study. One family CCF participant did not agree to give consent on the consent form, but proceeded with the survey to completion. If the participant truly did not consent to participation, he or she could have withdrawn from the survey without submitting the responses. Therefore, in order to maximize the sample size and power for the study, this response was included in the statistical analysis.

\section{Descriptive statistics}

The percentage of participants who were certified Early Childhood Educators was determined to be $44.4 \%$ of all the CCFs that participated in the survey (Figure 1).

In regards to years of experience, $62.2 \%$ of the 45 CCFs surveyed were found to be working in a daycare setting for more than ten years (Figure 2).

The majority of the CCFs $(95.6 \%)$ had a sanitation plan in place that included the disinfection of toys. Two CCFs (4.4\%) responded that their daycare did not have a sanitation plan in place (Figure 3).

The percentage of CCFs that supplied soft toys to play with was determined to be $66.7 \%$ of all the CCFs that participated in the survey as shown in Figure 4.
Figure 1. Certified Early Childhood Educators

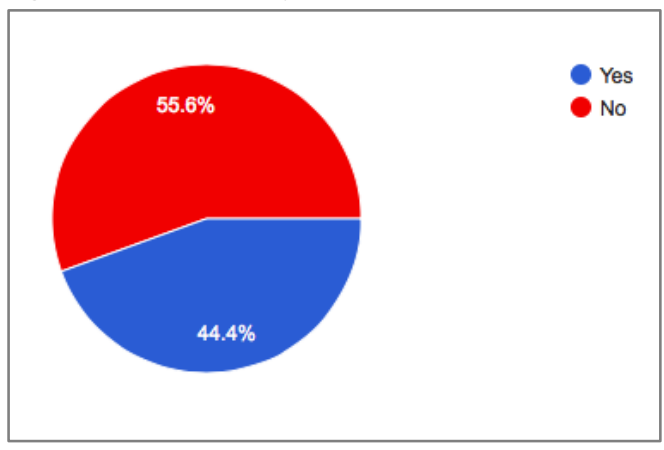

Figure 2. Years of experience in daycare setting

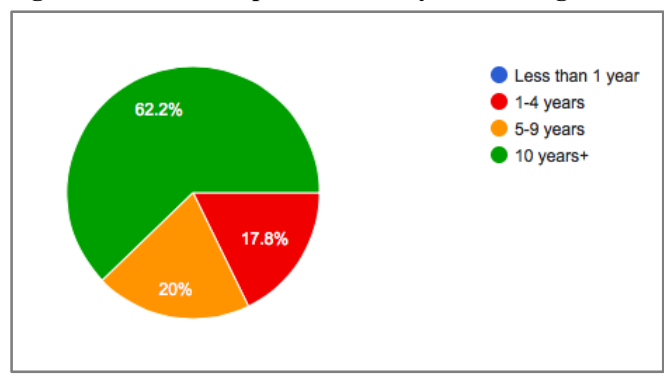

Figure 3. Presence of sanitation plan in place

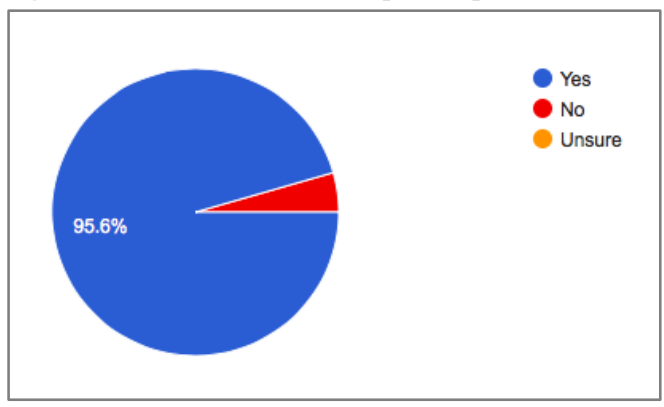

Figure 4. Soft toys present in CCFs

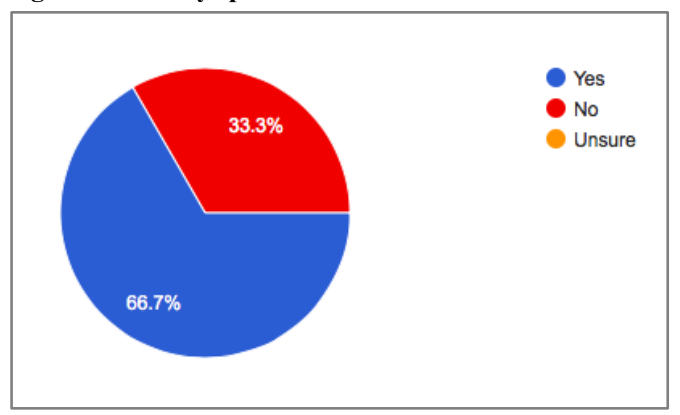

The responses of CCFs regarding how often toys are cleaned and sanitized were highly varied. The majority of the CCFs (84.4\%) responded that toys are cleaned and sanitized at least once a week. Two CCFs $(4.4 \%)$ responded that their toys were rarely or never cleaned and sanitized and one CCF (2.2\%) was unsure of the frequency of cleaning and sanitizing toys (Figure 5). 
Figure 5. Frequency of cleaning and sanitizing toys

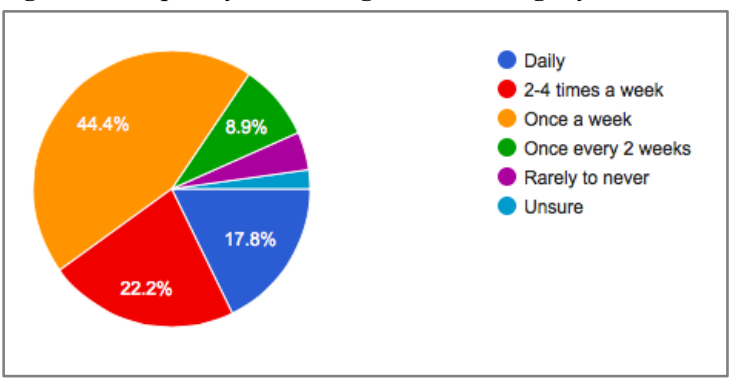

Figure 6 shows that there is a varied response rate for the correct sequence of actions for proper sanitation of surfaces. As children may put toys in their mouths, toys are considered food contact surfaces that should be sanitized in the following sequence: Clean, rinse, sanitize, and dry $(24,25)$. $46.7 \%$ of the CCFs surveyed answered the correct sequence of actions.

Based on results of previous studies, soft toys are more likely to be contaminated with bacteria than hard toys (26). The data shows that $68.9 \%$ of CCFs answered the question correctly (Figure 7).

Hard toys can be sanitized by the following methods: Sanitizing cycle of a dishwasher, bleach and water, disinfectant, and commercial disinfecting wipes (21). The answer is selecting all four methods. Although the responses varied, $93.3 \%$ of the CCFs responded that using bleach and water was a possible way to sanitize hard toys (Figure 8).

Soft toys can be cleaned and sanitized in a washing machine and dried in the dryer on normal settings (21). $73.3 \%$ of the CCFs had correct responses (Figure 9).

Previous studies have shown that natural products, such as vinegar, baking soda, and tea tree oil, are not effective disinfectants for food contact surfaces (27). As mentioned previously, toys are also considered food contact surfaces, and therefore, these products should not be used to clean toys (24). 53.3\% of the CCFs responded correctly (Figure 10).

Figure 6. Responses to the order of proper sanitation

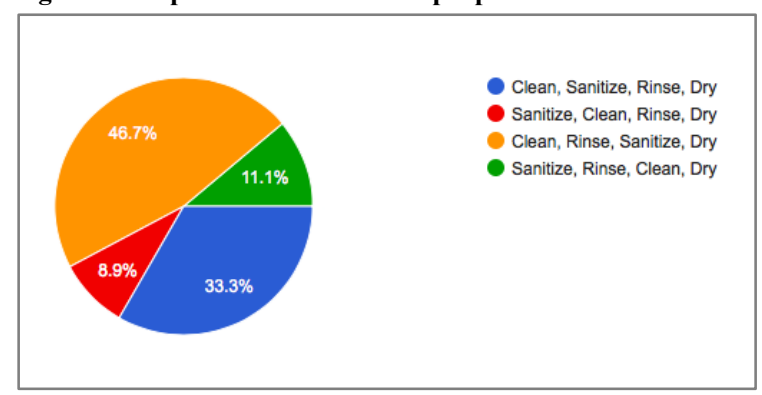

Figure 7. Responses to whether soft toys are more likely to be contaminated with bacteria than hard toys

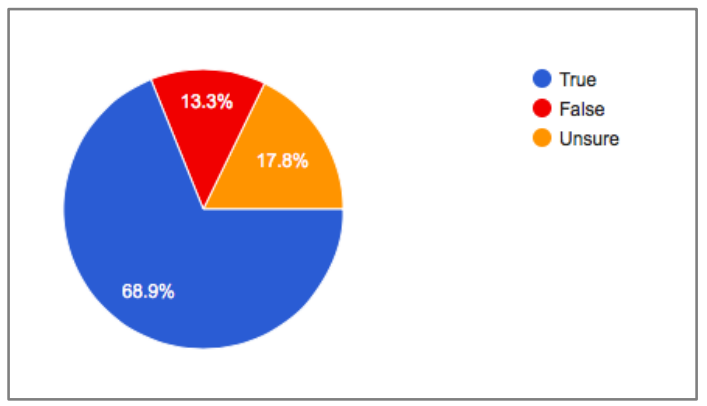

Figure 8. Responses to selecting all possible ways to sanitize hard toys

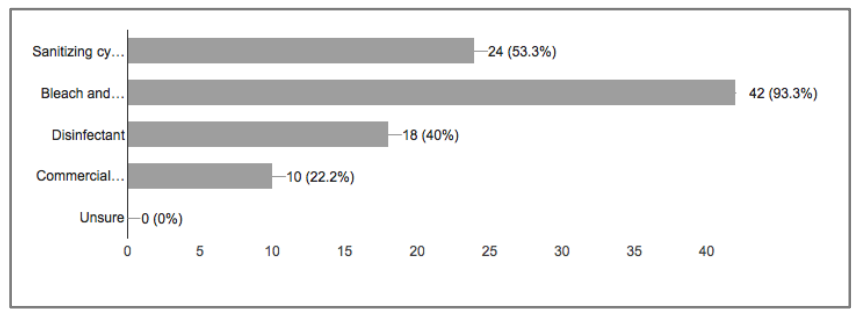

Responses in descending order: Sanitizing cycle in dishwasher, Bleach and water, Disinfectant, Commercial disinfecting wipes

Figure 9. Responses to whether soft toys can be cleaned and sanitized in a washing machine

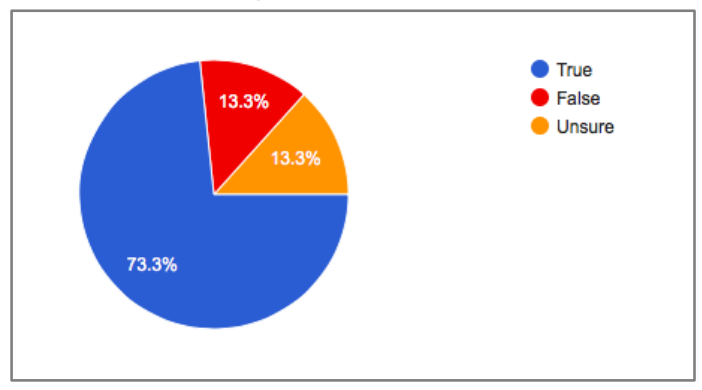

Figure 10. Responses to whether natural products are effective disinfectants that can be used for daycare toys

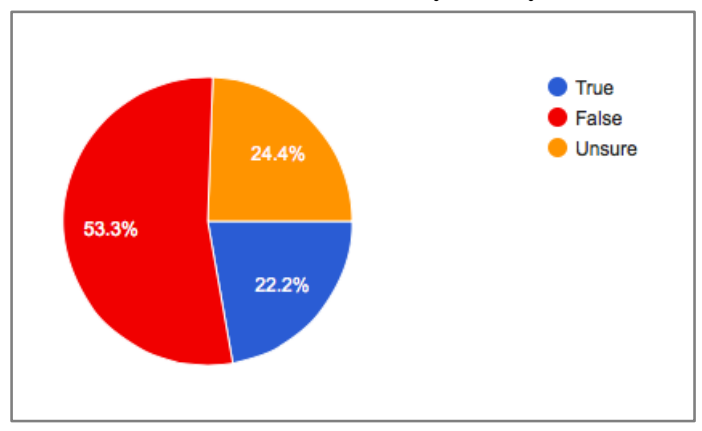

\section{Inferential statistics}

Each answer to the knowledge assessment survey questions was scored with a numerical value and each $\mathrm{CCF}$ that participated in the survey received a score out of 6 . These numbers were used to conduct an 
independent samples t-test to compare the difference in knowledge on sanitation of toys between group and family CCFs. The hypothesis for this test is as follows:

$H_{0}$ : There is no difference in the knowledge on sanitation of toys between group and family CCFs. $H_{1}$ : There is a difference in the knowledge on sanitation of toys between group and family CCFs.

The descriptive statistics shows that the mean score of group and family CCFs is 3.9 (65\%) and 3.3 (55\%) respectively. The standard deviation of group and family CCFs is 1.23 and 1.16 respectively.

The p-value from the Mann-Whitney $\mathrm{U}$ test is 0.088512 , which is greater than 0.05 , and thus, the null hypothesis is not rejected. It can be concluded that there is no statistically significant difference between the knowledge of sanitation of toys between group and family CCFs.

\section{Discussion}

The results of this study indicated that there was no difference between the knowledge of sanitation of toys between group and family CCFs. Although the mean score of the knowledge assessment of group CCFs (65\%) was slightly higher than family CCFs (55\%), it is important to note that the mean scores for both types of CCFs were below $67 \%$. The results may suggest that there is no gap between any prior education received as group or family childcare providers. However, there is significant room for improvement on the knowledge of sanitation of toys for both CCFs.

For the knowledge assessment portion of the survey, specific questions and responses raised environmental and public health concerns regarding both CCFs. 43 (95.6\%) out of 45 CCFs that were surveyed responded that their facility had a sanitation plan in place that included the disinfection of toys. However, the responses regarding the correct sequence of proper sanitation of surfaces including toys were highly varied. The correct answer was selected by 21 (46.7\%) of the CCFs. Utilizing incorrect procedures of sanitation may not be effective in removing contaminants or pathogens that cause infectious diseases from toys. Shared toys can have a major role in indirect transmission of disease, as they can be a reservoir for pathogens in saliva, feces, and other respiratory secretions (28). Also, children tend to put toys into their mouths, so toys should be disinfected as a food contact surface (24). It can be argued that there is a potential health hazard posed when toys are not disinfected properly and thus, there is an underlying concern when less than the majority of CCFs surveyed did not know correct sanitation procedures.

In addition, $10(22.2 \%)$ of the CCFs responded that natural products are effective disinfectants that can be used for daycare toys and 11 (24.4\%) CCFs were unsure. Previous studies have shown that natural products, such as vinegar, baking soda, and tea tree oil, have qualities that limit the application of these products as disinfectants on hard surfaces (29). Also, as mentioned above, toys are considered food contact surfaces, so they should be disinfected using food contact surface disinfectants approved by Health Canada $(24,29)$. Gaulin et al. have reported that natural products are not registered as food contact surface disinfectants by Health Canada (27). Therefore, natural products should not be used in order to effectively disinfect toys. Using ineffective disinfectants for toys may increase the potential for infectious diseases within CCFs, and thus, it is necessary to acknowledge the lack of knowledge of using proper disinfectants for toys.

\section{Recommendations}

Due to lack of standards and legislation regarding sanitation of CCFs, childcare providers depend on their own knowledge to provide clean, sanitary environments and toys for children. Results of this study suggest that there is a necessity for a set of standards or guidelines that CCFs can refer to for proper sanitation of toys.

In addition to standards or guidelines, if time and resources permit, implementing a continuing education credit requirement of CCFs could ensure that knowledge of all childcare providers is current. The continuing education credits could be obtained by attending educational programs on general sanitation and infection prevention and control for childcare providers. This program could be coordinated with childcare licensing officers or environmental health officers from various health authorities.

\section{Limitations}

The survey was distributed online and was highly dependent on honesty and accuracy of the answers of 
participants. This method cannot ensure that the survey is administered in a consistent manner between all participants, which limits validity. Reliability is also limited as participants could change their answers or search for correct answers using other resources. If time permitted, an in-person survey would have increased reliability and validity of the study, as questions would have been administered consistently and responses would have been immediate.

A total of 181 CCFs were called and 45 CCFs participated. The lack of participation in surveys limited the sample size and validity. The majority of CCFs that did not participate were unresponsive to phone calls that were made in the morning, afternoon, and evening on separate occasions. Some CCFs did not participate due to lack of technology, such as computers and e-mail accounts, and others did not participate due to lack of time to participate. A subset of CCFs chose not to participate, because they did not want to disclose any information despite the guarantee of confidentiality.

A lack of time and money limited the extent of the study. Time restraints resulted in a limited sample size of 45 CCFs, restricted only to CCFs in Surrey, BC. A larger sample size would have increased validity and further confirmed the results. Selecting CCFs from a more representative population, such as all of Lower Mainland or the Metro Vancouver region, would have resulted in a larger sample size as well. Additional monetary funds could have been used to conduct other studies to support the results, such as quantifying the amount of microbes or coliforms present on toys of CCFs that participated in the surveys. Previous studies have reflected the value of microbiological sampling of toys. In 2010, Jang's study (30) indicated that there was a statistically significant difference between aerobic bacterial counts on hard toys in comparison to countertops in general practitioner's offices in Vancouver, with an elevated count on the toys. The study had concluded that the potential of disease transmission through toys as a fomite is at large.

However, Chiu's study (31) found no bacteria pre- and post-cleaning daycare toys at a group daycare located in Simon Fraser University. If this daycare is representative of all group daycares, daycare toys may not be a risky fomite for disease transmission, contrary to existing literature.

\section{Future Research}

Based on the results of this study, further research areas may be pursued. For a better representative of knowledge of sanitation of toys, similar research studies may be conducted by surveying group and family CCFs of other regions. In addition, to analyze if there is an association with knowledge of general sanitation or sanitation of toys and infectious diseases, future studies could involve obtaining sickness or absence data of children from CCFs.

\section{Conclusions}

This research study showed that there was no difference between group and family CCFs on knowledge of sanitation of toys. However, a significant lack of knowledge was still observed from both types of CCFs. Children are vulnerable populations that are susceptible to infectious diseases, and thus, knowledge deficits on proper cleaning and sanitation of toys is a public health concern. Emphasis should be put on educating childcare providers for proper infection control of toys, which can be reservoirs for infectious pathogens. Due to various limitations and the nature of this study, further research with higher reliability and validity is needed to confirm the results.

\section{Acknowledgements}

This research was completed with the support of supervisors Helen Heacock and Vanessa Karakilic as well as the Environmental Health Program at the British Columbia Institute of Technology.

\section{Competing interest}

The author declares that there are no competing interests. 


\section{References}

(1) Statistics Canada. Children and Youth

Research Paper Series: Child Care in

Canada [Internet]. 2006. Available from:

http://www.statcan.gc.ca/pub/89-599-m/89-

599-m2006003-eng.pdf

(2) Pijnacker R, Mughini-Gras L, Vennema H, Enserink R, Van Den Wijngaard CC, Kortbeek T, et al. Characteristics of child daycare centres associated with clustering of major enteropathogens. Epidemiol Infect. 2016;144:2527-39.

(3) Herman D. Day-Care Centres: Risks and Prevention of Infection. Can Fam Physician. 1988;34:1191-5.

(4) Little K, Cutcliffe S. The safe use of children's toys within the healthcare setting. Nurs times [Internet]. 2006;102(38):34-7. Available from: http://www.scopus.com/inward/record.url?ei $\mathrm{d}=2$-s2.0$33750579651 \&$ partnerID $=40 \& \mathrm{md} 5=92 \mathrm{~d} 6 \mathrm{c} 4$ $16 \mathrm{e} 35 \mathrm{acfff} 47 \mathrm{c} 1 \mathrm{c} 8683899 \mathrm{a} 711$

(5) Van R, Morrow AL, Reves RR, Pickering LK. Environmental contamination in child day-care centers. Am J Epidemiol [Internet]. 1991;133(5):460-70. Available from: http://www.ncbi.nlm.nih.gov/pubmed/20008 56

(6) Enserink R, Mughini-Gras L, Duizer E, Kortbeek T, Van Pelt W. Risk factors for gastroenteritis in child day care. Epidemiol Infect [Internet]. 2015;143(13):2707-20. Available from: http://journals.cambridge.org/abstract_S095 0268814003367

(7) Ibfelt T, Engelund EH, Schultz AC, Andersen LP. Effect of cleaning and disinfection of toys on infectious diseases and micro-organisms in daycare nurseries. $\mathrm{J}$ Hosp Infect. 2015;89:109-15.

(8) Martin ET, Fairchok MP, Stednick ZJ, Kuypers J, Englund JA. Epidemiology of multiple respiratory viruses in childcare attendees. J Infect Dis. 2013;207(6):982-9.

(9) Carabin H, Gyorkos TW, Soto JC, Joseph L, Payment P, Collet JP. Effectiveness of a training program in reducing infections in toddlers attending day care centers. Epidemiology [Internet]. 1999;10(3):21927. Available from: http://www.ncbi.nlm.nih.gov/pubmed/10230 828

Lee L, Tin S, Kelley ST. Cultureindependent analysis of bacterial diversity in a child-care facility. BMC Microbiol [Internet]. 2007;7(27). Available from: http://www.biomedcentral.com/1471$2180 / 7 / 27$

Pickering LK, Bartlett AV, Woodward WE. Acute infectious diarrhea among children in day care: epidemiology and control. Rev Infect Dis [Internet]. 1986;8(4):539-47. Available from: http://www.ncbi.nlm.nih.gov/pubmed/35293 10

Wilde J, Van R, Pickering L, Eiden J, Yolken R. Detection of rotaviruses in the day care environment by reverse transcriptase polymerase chain reaction. J Infect Dis. 1992;166(0022-1899 (Print)):507-11.

Fraser A, Wohlgenant K, Cates S, Chen X, Jaykus LA, Li Y, et al. An observational study of frequency of provider hand contacts in child care facilities in North Carolina and South Carolina. Am J Infect Control. 2015;43(2):107-11.

Holaday B, Pantell R, Lewis C, Gilliss CL. Patterns of fecal coliform contamination in day-care centers. Public Health Nurs [Internet]. 1990;7(4):224-8. Available from: https://www.ncbi.nlm.nih.gov/pubmed/2270 220

Ibfelt T, Engelund E, Andersen L. P248: Can hand hygiene and cleaning reduce the number of sick days in day care centers? Antimicrob Resist Infect Control. 2013;2(Suppl 1):25-8.

Sauver JS, Khurana M, Kao A, Foxman B. Hygienic practices and acute respiratory illness in family and group day care homes. Public Health Rep. 1998;113(6):544-51.

Rogers M, Weinstock DM, Eagan J, Kiehn T, Armstrong D, Sepkowitz KA. Rotavirus outbreak on a pediatric ontology floor: 
Possible association with toys. Am J Infect

Control. 2000;28(5):378-80.

(18) Cosby CM, Costello CA, Morris WC, Haughton B, Devereaux MJ, Harte F, et al. Microbiological analysis of food contact surfaces in child care centers. Appl Environ Microbiol. 2008;74(22):6918-22.

(19) Child Care Licensing Regulation. BC Reg 332/2007

(20) Community Care and Assisted Living Act, 2010, SBC 2002, c 75.

(21) Vancouver Coastal Health. Sneezes and diseases: A resource book for caregivers and parents. [Internet]. Vancouver: Vancouver Coastal Health; 2014 [cited 2017 Mar 9]. Available from: http://www.vch.ca/media/Sneezes_And_Dis eases_December_2014.pdf

(22) Fraser Health, Health Protection, Community Care Facilities Licensing Program. Public list of licensed child care facilities: Fraser South (Health Service Delivery Area \#23). [Internet]. Surrey: Fraser Health; 2016 [cited 2016 Nov 20]. Available from: http://www.fraserhealth.ca/media/201609_C hildCareFacilities_SOUTH.pdf

(23) Heacock, H. ENVH 8400 Research Methods Module 4: Research design and data collection. Lecture presented at; 2016; BCIT.

(24) Missouri Department of Health and Senior Services. Environmental health operational guidelines. [Internet]. Jefferson City:

Missouri Department of Health and Senior Services; 2008 [cited 2017 Mar 9].

Available from: http://health.mo.gov/atoz/ehog/pdf/Ch_4.1.6 .pdf

(25) Alberta Health Services, Environmental Public Health. Health and safety guidelines for child care facilities. [Internet]. Edmonton: Alberta Health Services; 2014 [cited 2017 March 9]. Available from: http://www.albertahealthservices.ca/assets/w f/eph/wf-eh-health-safety-guidlines-childcare-facilities.pdf
(26) Merriman E, Corwin P, Ikram R. Toys are a potential source of cross-infection in general practitioners' waiting rooms. Br J Gen Pract. 2002;52:138-40.

(27) Gaulin C, Lê M-L, Shum M, Fong D. Disinfectants and sanitizers for use on food contact surfaces. [Internet]. Vancouver: National Collaborating Centre for Environmental Health; 2011 [cited 2017 Mar 9]. Available from: http://www.ncceh.ca/sites/default/files/Food _Contact_Surface_Sanitizers_Aug_2011.pdf

Infection Prevention and Control (IPAC) Canada, IPAC Canada's Paediatric and Neonatal Interest Group. IPAC Canada practice recommendations for toys. [Internet]. Winnipeg: IPAC-Canada; 2011 [cited 2017 Mar 9]. Available from: http://ipaccanada.org/photos/custom/OldSite/pdf/Toys $\% 20$ Practice $\% 20$ Recommendations $\% 20201$ 1\%20-\%20R2014.pdf

Fong D, Gaulin C, Lê M-L, Shum M. Effectiveness of alternate antimicrobial agents for disinfection of hard surfaces. [Internet]. Vancouver: National Collaborating Centre for Environmental Health; 2011 [cited 2017 Mar 9]. Available from:

http://www.ncceh.ca/sites/default/files/Food _Contact_Surface_Sanitizers_Aug_2011.pdf

Jang K. An evaluation of infection control measures on hard toys in general practitioners' offices in Vancouver BC Canada using microbiological sampling. Vancouver: British Columbia Institute of Technology; 2010 (Unpublished).

Chiu J. Mean difference of coliform counts in relation to sanitation frequencies at the Simon Fraser University Childcare Society. Vancouver: British Columbia Institute of Technology; 2017 (Unpublished). 